TRANSACTIONS OF THE

AMERICAN MATHEMATICAL SOCIETY

Volume 358, Number 8, Pages 3717-3731

S 0002-9947(06)03862-1

Article electronically published on March 24, 2006

\title{
LENGTH, MULTIPLICITY, AND MULTIPLIER IDEALS
}

\author{
TOMMASO DE FERNEX
}

\begin{abstract}
Let $(R, \mathfrak{m})$ be an $n$-dimensional regular local ring, essentially of finite type over a field of characteristic zero. Given an m-primary ideal $\mathfrak{a}$ of $R$, the relationship between the singularities of the scheme defined by $\mathfrak{a}$ and those defined by the multiplier ideals $\mathcal{J}\left(\mathfrak{a}^{c}\right)$, with $c$ varying in $\mathbb{Q}_{+}$, are quantified in this paper by showing that the Samuel multiplicity of $\mathfrak{a}$ satisfies $e(\mathfrak{a}) \geq(n+k)^{n} / c^{n}$ whenever $\mathcal{J}\left(\mathfrak{a}^{c}\right) \subseteq \mathfrak{m}^{k+1}$. This formula generalizes an inequality on $\log$ canonical thresholds previously obtained by Ein, Mustaţă and the author of this paper. A refined inequality is also shown to hold for small dimensions, and similar results valid for a generalization of test ideals in positive characteristics are presented.
\end{abstract}

\section{INTRODUCTION}

Let $\mathfrak{a}$ be an ideal of an $n$-dimensional regular local ring $(R, \mathfrak{m})$ essentially of finite type over a field of characteristic zero. Consider a log resolution of $(R, \mathfrak{a})$, namely, a proper birational morphism $f$ from a smooth variety $Y$ to $\operatorname{Spec} R$ such that $f^{-1} \mathfrak{a} \cdot \mathcal{O}_{Y}$ is the ideal sheaf of a simple normal crossing divisor $E$ on $Y$. Then, for any positive rational number $c$, one defines the multiplier ideal associated to the pair $\left(R, \mathfrak{a}^{c}\right)$ to be the ideal

$$
\mathcal{I}\left(\mathfrak{a}^{c}\right):=f_{*} \mathcal{O}_{Y}\left(K_{f}-[c E]\right) \subseteq R,
$$

where $K_{f}$ is the relative canonical divisor of $f$ and $[c E]$ is the integral part of the $\mathbb{Q}$-divisor $c E$. This ideal is a fundamental invariant of the singularity of the scheme defined by $\mathfrak{a}$, and its nice properties related to vanishing theorems make it an important tool in higher-dimensional geometry. For general properties of multiplier ideals, we refer to $\mathrm{Laz}$.

For different values of $c$, the multiplier ideals $\mathcal{I}\left(\mathfrak{a}^{c}\right)$ offer a way to look at the singularities of the subscheme defined by $\mathfrak{a}$. The goal of this paper is to quantify this principle assuming that $\mathfrak{a}$ is $\mathfrak{m}$-primary, by reading off conditions on the Samuel multiplicity $e(\mathfrak{a})$ and the co-length $\ell(R / \mathfrak{a})$ of $\mathfrak{a}$.

Since the Samuel multiplicity is homogeneous with respect to integer powers of $\mathfrak{m}$-primary ideals, we can set $e\left(\mathfrak{a}^{c}\right):=c^{n} e(\mathfrak{a})$ for any $\mathfrak{m}$-primary ideal $\mathfrak{a}$ of $R$ and any $c \in \mathbb{Q}_{+}$. Although this definition is not strictly necessary, it has the advantage

Received by the editors June 21, 2004 and, in revised form, September 20, 2004.

2000 Mathematics Subject Classification. Primary 14B05; Secondary 13H05, 14B07, 13 H15.

Key words and phrases. Multiplier ideal, Samuel multiplicity, monomial ideal.

The author's research was partially supported by the University of Michigan Rackham Research Grant and Summer Fellowship, and by the MIUR of the Italian Government in the framework of the National Research Project "Geometry on Algebraic Varieties" (Cofin 2002).

(C)2006 American Mathematical Society Reverts to public domain 28 years from publication 
of making the statement below more symmetric and, we hope, transparent. Using the notation just introduced and observing that $e\left(\mathfrak{m}^{n}\right)=n^{n}$, the main result from dFEM1] (namely, Theorem 1.2), that was originally stated in terms of the $\log$ canonical threshold of the pair $(R, \mathfrak{a})$, can be reformulated as follows:

$$
e\left(\mathfrak{a}^{c}\right) \geq e\left(\mathfrak{m}^{n}\right) \quad \text { whenever } \quad \mathcal{I}\left(\mathfrak{a}^{c}\right) \subseteq \mathfrak{m} .
$$

It is natural to expect a stronger bound on $e\left(\mathfrak{a}^{c}\right)$ when the multiplier ideal $\mathcal{I}\left(\mathfrak{a}^{c}\right)$ is strictly smaller than $\mathfrak{m}$. This motivated us towards the next result.

Theorem 0.1. Let $R$ be as above, and let $\mathfrak{a}$ be an $\mathfrak{m}$-primary ideal $R$. Then, for any rational $c>0$ and any integer $k \geq 0$, we have

$$
e\left(\mathfrak{a}^{c}\right) \geq e\left(\mathfrak{m}^{n+k}\right) \quad \text { whenever } \quad \mathcal{I}\left(\mathfrak{a}^{c}\right) \subseteq \mathfrak{m}^{k+1} .
$$

The lower bound on the multiplicity given in this theorem is sharp, and the boundary cases are characterized: equality occurs exactly when $(n+k) / c$ is an integer and the integral closure of $\mathfrak{a}$ is equal to $\mathfrak{m}^{(n+k) / c}$ (see Theorem 2.3 below).

Theorem 0.1 is derived by a similar result in which the length of $R / \mathfrak{a}$ is bounded, instead of the multiplicity of $\mathfrak{a}$ (this is Theorem 2.1 below). Moreover, if the dimension of $R$ is at most 3 and $c=1$, the result on the length is, in turn, implied by the following general formula.

Theorem 0.2. Let $R$ be a ring as above, and assume that $R$ has dimension $n \leq 3$. Let $\mathfrak{a}$ be an $\mathfrak{m}$-primary ideal of $R$ such that $\mathcal{I}(\mathfrak{a})$ is not trivial. Then

$$
\ell(R / \mathfrak{a}) \geq \ell\left(R / \mathcal{I}(\mathfrak{a}) \mathfrak{m}^{n-1}\right) .
$$

These results have analogues in positive characteristic. In [HY], Hara and Yoshida have introduced a new ideal associated to a pair $\left(R, \mathfrak{a}^{c}\right)$ when the ground field has positive characteristic. Such ideal is denoted by $\tau\left(\mathfrak{a}^{c}\right)$; it is a generalization of the test ideal defined by Hockster and Huneke, and can be considered as the analogue of the multiplier ideal. In the last section of this paper we will explain how the main theorems of this paper, stated for multiplier ideals in characteristic zero, also hold for this new ideal when the characteristic is positive. The idea comes from Takagi and Watanabe's paper [TW], where the F-pure threshold, a positive characteristic analogue of the log canonical threshold, is defined. Indeed it was observed in [TW that the same bound as the one established for log canonical thresholds in dFEM1, Theorem 1.2, also holds for the F-pure threshold.

\section{BASICS ON MONOMIAL IDEALS}

In this paper, the set of natural numbers $\mathbb{N}$ includes zero. For short, we denote by $\mathbb{R}_{+}^{n}$ the set $\left(\mathbb{R}_{\geq 0}\right)^{n}$, and view it as a subset of the vector space $\mathbb{R}^{n}$. Similarly, we view $\mathbb{N}^{n}$ as a subset of $\mathbb{R}_{+}^{n}$. We denote by $\operatorname{Vol}(S)$ the volume of a measurable subset $S$ of $\mathbb{R}^{n}$. The interior $\operatorname{Int}(S)$ of a subset $S \subset \mathbb{R}^{n}$ will always be computed in $\mathbb{R}^{n}$ (even if $S$ is given as a subset of $\mathbb{R}_{+}^{n}$ ). For a vector $\mathbf{u}=\left(u_{i}\right) \in \mathbb{R}^{n}$, we denote by $|\mathbf{u}|$ its one-norm, that is, $|\mathbf{u}|=\sum\left|u_{i}\right|$. Given a hyperplane $H$ defined in $\mathbb{R}^{n}$ by an equation of the form $\sum b_{i} u_{i}=1$ with $b_{i} \in \mathbb{R}$, we introduce the following notation:

$$
H^{+}=\left\{\mathbf{u} \in \mathbb{R}_{+}^{n} \mid \sum b_{i} u_{i} \geq 1\right\}, \quad H^{-}=\left\{\mathbf{u} \in \mathbb{R}_{+}^{n} \mid \sum b_{i} u_{i} \leq 1\right\} .
$$

Let $\left\{\mathbf{e}_{1}, \ldots, \mathbf{e}_{n}\right\}$ be the standard basis of $\mathbb{R}^{n}$, and set $\mathbf{e}=\sum \mathbf{e}_{i}$.

Let $k$ be a field, let $R=k\left[x_{1}, \ldots, x_{n}\right]$, and denote $\mathfrak{m}=\left(x_{1}, \ldots, x_{n}\right)$. To a monomial $\prod x_{i}^{u_{i}} \in R$, we associate the vector $\mathbf{u}=\left(u_{i}\right) \in \mathbb{N}^{n}$ (we will also use the 
shorter notation $\mathbf{x}^{\mathbf{u}}$ to denote the monomial). The Newton polygon $P(\mathfrak{a})$ associated to a monomial ideal $\mathfrak{a} \subset R$ is, by definition, the convex hull in $\mathbb{R}_{+}^{n}$ of the vectors that correspond in this fashion to the monomials in $\mathfrak{a}$. Given a monomial ideal $\mathfrak{a}$ and a positive integer $r$, we have $P\left(\mathfrak{a}^{r}\right)=r P(\mathfrak{a})$. It is natural then to set $P\left(\mathfrak{a}^{c}\right):=c P(\mathfrak{a})$ for any positive rational number $c$. Note that

$$
\operatorname{Vol}\left(\mathbb{R}_{+}^{n} \backslash P\left(\mathfrak{a}^{c}\right)\right)=c^{n} \operatorname{Vol}\left(\mathbb{R}_{+}^{n} \backslash P(\mathfrak{a})\right) .
$$

We will use the basic property saying that, for an $\mathfrak{m}$-primary monomial ideal $\mathfrak{a}$ of $R$,

$$
\ell(R / \mathfrak{a}) \geq \operatorname{Vol}\left(\mathbb{R}_{+}^{n} \backslash P(\mathfrak{a})\right),
$$

and the inequality is strict if $n \geq 2$; for a proof, see for instance [FEM1], Lemma 1.3. We will also use the characterization of Samuel multiplicity of monomial ideals that says that

$$
e(\mathfrak{a})=n ! \operatorname{Vol}\left(\mathbb{R}_{+}^{n} \backslash P(\mathfrak{a})\right)
$$

for any $\mathfrak{m}$-primary monomial ideal $\mathfrak{a}$ of $R$ (see Tei], page 131).

For the reminder of this section, we assume that the field $k$ has characteristic zero.

Theorem 1.1 ([How, Main Theorem). Let $\mathfrak{a}$ be a monomial ideal of a polynomial ring $k\left[x_{1}, \ldots, x_{n}\right]$, where $k$ is a field of characteristic zero, and let $c \in \mathbb{Q}_{+}$. Then $\mathcal{I}\left(\mathfrak{a}^{c}\right)$ is a monomial ideal as well, and $\mathbf{x}^{\mathbf{u}} \in \mathcal{I}\left(\mathfrak{a}^{c}\right)$ if and only if $\mathbf{u}+\mathbf{e} \in \operatorname{Int}\left(P\left(\mathfrak{a}^{c}\right)\right) \cap$ $\mathbb{N}^{n}$.

By suitably fixing a monomial order on a polynomial ring $R=k\left[x_{1}, \ldots, x_{n}\right]$, we can define a flat deformation of an ideal $\mathfrak{a}$ of $R$ to its initial ideal in(a) (this is well explained in Eis, Section 15.8). We can additionally assume that, for a given $c$, this simultaneously gives a flat deformation of $\mathcal{I}\left(\mathfrak{a}^{c}\right)$ to its initial ideal in $\left(\mathcal{I}\left(\mathfrak{a}^{c}\right)\right)$. Given this situation, we will use the following property; as we will need to discuss an analogous property in positive characteristic (see Proposition 5.3 below), we include the proof for reference.

Proposition 1.2 (dFEM2, Lemma 2.3). Let $\mathfrak{a}$ be an ideal of a polynomial ring $R=k\left[x_{1}, \ldots, x_{n}\right]$, where $k$ is a field of characteristic zero, and consider a flat deformation to initial ideals as described above. Then

$$
\mathcal{I}\left(\operatorname{in}(\mathfrak{a})^{c}\right) \subseteq \operatorname{in}\left(\mathcal{I}\left(\mathfrak{a}^{c}\right)\right) .
$$

Proof. Let $t$ be the parameter of deformation for the degeneration to monomial ideals fixed above. Let $T=k\left[x_{1}, \ldots, x_{n}, t\right]$, and let $\mathfrak{b} \subset T$ be the ideal corresponding to the deformation of $\mathfrak{a}$. There is an isomorphism from $T_{t}:=k\left[x_{1}, \ldots, x_{n}, t, t^{-1}\right]$ to $R \otimes k\left[t, t^{-1}\right]$ sending $\mathfrak{b} T_{t}$ to $\mathfrak{a} \otimes k\left[t, t^{-1}\right]$. Via this isomorphism, we have $\mathcal{I}\left(\left(\mathfrak{b} T_{t}\right)^{c}\right) \cong$ $\mathcal{I}\left(\mathfrak{a}^{c}\right) \otimes k\left[t, t^{-1}\right]$. Since the family degenerating to the initial ideal is flat, we have

$$
\mathcal{I}\left(\mathfrak{b}^{c}\right) /\left((t) \cap \mathcal{I}\left(\mathfrak{b}^{c}\right)\right) \subseteq \operatorname{in}\left(\mathcal{J}\left(\mathfrak{a}^{c}\right)\right)
$$

via the canonical isomorphism $T /(t) \cong R$. On the other hand, by standard properties of multiplier ideals (see [Laz, Section 9.5.A), we have

$$
\mathcal{I}\left(\operatorname{in}(\mathfrak{a})^{c}\right)=\mathcal{I}\left((\mathfrak{b} /((t) \cap \mathfrak{b}))^{c}\right) \subseteq \mathcal{I}\left(\mathfrak{b}^{c}\right) /\left((t) \cap \mathcal{I}\left(\mathfrak{b}^{c}\right)\right) .
$$

If we put together the above inclusions, we get the assertion of the proposition. 


\section{LOWER BOUNDS TO LENGTH AND MULTIPLICITY}

Throughout this section, $(R, \mathfrak{m})$ is an $n$-dimensional regular local ring, essentially of finite type over a field of characteristic zero. We begin with the following result on the co-length of an m-primary ideal of $R$.

Theorem 2.1. Let $\mathfrak{a}$ be an $\mathfrak{m}$-primary ideal of $R$. Fix $c \in \mathbb{Q}_{+}$and $k \in \mathbb{N}$, and assume that $\mathcal{I}\left(\mathfrak{a}^{c}\right) \subseteq \mathfrak{m}^{k+1}$. Then

$$
\ell(R / \mathfrak{a}) \geq \frac{(n+k)^{n}}{n ! c^{n}},
$$

and the inequality is strict if $n \geq 2$.

We apply the above result to prove the first theorem stated in the Introduction, that we reformulate as follows.

Theorem 2.2. With assumptions as in Theorem 2.1, we have

$$
e(\mathfrak{a}) \geq \frac{(n+k)^{n}}{c^{n}} .
$$

Proof. By applying Theorem 2.1 to $\mathfrak{a}^{m}$ and $c / m$ in place of $\mathfrak{a}$ and $c$ in the following limit, we get

$$
e(\mathfrak{a})=\lim _{m \rightarrow \infty} \frac{\ell\left(R / \mathfrak{a}^{m}\right)}{\left(m^{n} / n !\right)} \geq \frac{(n+k)^{n}}{c^{n}} .
$$

It is immediate to check that the hypotheses of Theorem 2.2 are satisfied if the integral closure of $\mathfrak{a}$ is a power, say $\mathfrak{m}^{a}$, of the maximal ideal $\mathfrak{m}$, and $c=(n+k) / a$. Note that in this case the lower bound to the multiplicity given in (2.2) is achieved. It turns out that these are the only cases realizing the bound.

Theorem 2.3. Under the assumptions of Theorem 2.2, $e(\mathfrak{a})=(n+k)^{n} / c^{n}$ if and only if $(n+k) / c \in \mathbb{N}$ and $\overline{\mathfrak{a}}=\mathfrak{m}^{(n+k) / c}$.

The proofs of Theorems 2.1 and 2.3 are given in the next section. The cases $k=0$ in the three theorems above are precisely the main results obtained in dFEM1] (see, respectively, Theorems 1.1, 1.2 and 1.4 ibidem).

\section{Proofs of Theorem 2.1 and Theorem 2.3}

The proof of Theorem 2.1 is conceptually elementary. After reducing to the case of monomial ideals by a flat degeneration, we estimate the volume of the complement of the associated Newton polygon, and hence apply (1.2). The volume is opportunely bounded by replacing the above region by a new, smaller, symmetric region. Roughly speaking, the new region is constructed as the "average" of the original one under the action of the symmetric group on $\mathbb{R}_{+}^{n}$. A key point will be that the new region we construct is the complement (in $\mathbb{R}_{+}^{n}$ ) of a convex set.

Before passing to the proof of the theorem, we fix some notation and prove a preliminary property on convexity. Recall that $\mathbb{R}_{+}^{n}$ denotes the set $\left(\mathbb{R}_{\geq 0}\right)^{n}$, and it is viewed as a subset of $\mathbb{R}^{n}$. The span of any non-zero vector of $\mathbb{R}_{+}^{n}$ is called a ray of $\mathbb{R}_{+}^{n}$, and a connected bounded subset of a ray of $\mathbb{R}_{+}^{n}$ containing the origin of $\mathbb{R}_{+}^{n}$ is 
said to be a truncated ray. Given any two subsets $Q_{1}, Q_{2} \subseteq \mathbb{R}_{+}^{n}$ intersecting every ray of $\mathbb{R}_{+}^{n}$, we define their radial sum as the set

$$
Q_{1} \star Q_{2}:=\bigcup_{W}\left(\left(Q_{1} \cap W\right)+\left(Q_{2} \cap W\right)\right),
$$

where the union runs over all rays $W \subset \mathbb{R}_{+}^{n}$, and the sum appearing in the righthand side is the usual sum of two subsets of a vector space. To denote a radial summation, we will use the symbol $\star$. The following picture gives a graphical example of a radial sum of two segments $Q_{1}$ and $Q_{2}$ in $\mathbb{R}_{+}^{2}$ :

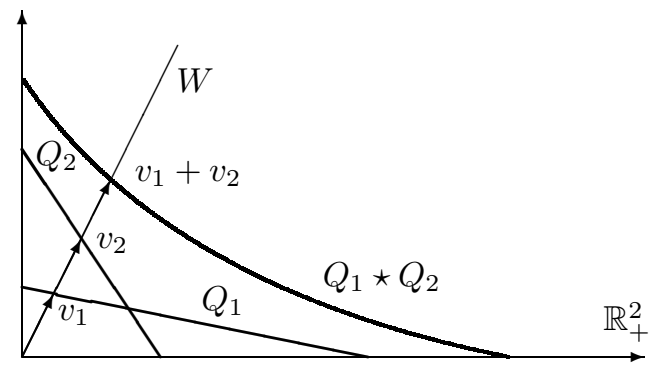

We will consider subsets $P \subset \mathbb{R}_{+}^{n}$ such that, for any ray $W \subset \mathbb{R}_{+}^{n}, W \backslash P$ is a truncated ray. To fix terminology, we will say that $P$ is a bounding subset of $\mathbb{R}_{+}^{n}$ if it is a subset satisfying the above property. Newton polygons associated to $\mathfrak{m}$-primary monomial ideals are examples of (closed and convex) bounding subsets of $\mathbb{R}_{+}^{n}$.

Proposition 3.1. If $P_{1}, P_{2} \subset \mathbb{R}_{+}^{n}$ are two closed, convex, bounding subsets of $\mathbb{R}_{+}^{n}$, then $P_{1} \star P_{2}$ is also a closed, convex, bounding subset of $\mathbb{R}_{+}^{n}$.

Proof. For any ray $W$, the set $W \backslash\left(P_{1} \star P_{2}\right)$ is a sum of two truncated rays supported by $W$, hence it is a truncated ray. In particular, $P_{1} \star P_{2}$ is a bounding subset of $\mathbb{R}_{+}^{n}$. It is also clear that $P_{1} \star P_{2}$ is closed. Then, to prove that $P_{1} \star P_{2}$ is convex, it is enough to show that it contains the segment joining any two distinct points on its boundary (boundaries are computed inside $\mathbb{R}_{+}^{n}$ ). Let $\mathbf{u}$ and $\mathbf{v}$ be two distinct vectors lying on the boundary of $P_{1} \star P_{2}$. Then $\mathbf{u}=\mathbf{u}_{1}+\mathbf{u}_{2}$ and $\mathbf{v}=\mathbf{v}_{1}+\mathbf{v}_{2}$ where, for each $i$, the vector $\mathbf{u}_{i}$ lies on the ray spanned by $\mathbf{u}$, the vector $\mathbf{v}_{i}$ lies on the ray spanned by $\mathbf{v}$, and both $\mathbf{u}_{i}$ and $\mathbf{v}_{i}$ are in the boundary of $P_{i}$. Since $P_{i}$ is convex, the segment joining $\mathbf{u}_{i}$ and $\mathbf{v}_{i}$ is contained in $P_{i}$. Moreover, since $P_{i}$ is a bounding subset of $\mathbb{R}_{+}^{n}$ and the two points are in its boundary, the line of $\mathbb{R}^{n}$ passing through $\mathbf{u}_{i}$ and $\mathbf{v}_{i}$ intersects $\mathbb{R}_{+}^{n}$ in a bounded segment. Note that the two segments obtained in this way (one for each value of $i$ ) lie on the two-dimensional subspace of $\mathbb{R}^{n}$ generated by $\mathbf{u}$ and $\mathbf{v}$. Then, after restricting to such a subspace, the convexity of $P_{1} \star P_{2}$ is manifestly established by the following property: For any two lines in $\mathbb{R}^{2}$ cutting two non-trivial and bounded segments $L_{1}$ and $L_{2}$ on $\mathbb{R}_{+}^{2}, L_{1} \star L_{2}$ is a curve with concavity towards the unbounded component of its complement in $\mathbb{R}_{+}^{2}$. (This property can be easily checked by parameterizing the points on each line by the slope of the ray they generate.)

Proof of Theorem 2.1. Multipliers ideals commute with localization and completion. Thus, by passing to completion, hence extending zero-dimensional ideals and 
localizing again, we can reduce to the case in which $R$ is the localization at the origin of a polynomial ring (for more details on the reduction, we refer to the proof of [dFEM1, Theorem 1.1). After fixing a monomial order on the coordinates, we obtain a flat degeneration of $\mathfrak{a}$ and $\mathcal{I}\left(\mathfrak{a}^{c}\right)$ to their initial ideals $\operatorname{in}(\mathfrak{a})$ and $\operatorname{in}\left(\mathcal{I}\left(\mathfrak{a}^{c}\right)\right)$. By flatness, we have $\ell(R / \mathfrak{a})=\ell(R /$ in $(\mathfrak{a}))$. On the other hand, using the hypothesis that $\mathcal{I}\left(\mathfrak{a}^{c}\right) \subseteq \mathfrak{m}^{k+1}$ together with Proposition 1.2, we get

$$
\mathcal{I}\left(\operatorname{in}(\mathfrak{a})^{c}\right) \subseteq \operatorname{in}\left(\mathcal{I}\left(\mathfrak{a}^{c}\right)\right) \subseteq \operatorname{in}\left(\mathfrak{m}^{k+1}\right)=\mathfrak{m}^{k+1} .
$$

Therefore, if we can prove the theorem for in $(\mathfrak{a})$, then we can deduce the bound for $\mathfrak{a}$. Thus we assume henceforth that $\mathfrak{a}$ is a monomial ideal.

Let us first discuss the special case in which $k$ is a multiple of $n$; as in this case the proof is very simple. We have $k=n q$ for some $q \in \mathbb{N}$. Since $|q \mathbf{e}|<k+1$, (3.1) implies that

$$
\mathbf{x}^{q \mathbf{e}} \notin \mathcal{I}\left(\mathfrak{a}^{c}\right) .
$$

Then $(q+1) \mathbf{e} \notin \operatorname{Int}\left(P\left(\mathfrak{a}^{c}\right)\right)$ by Theorem 1.1, hence $\mathbf{e} \notin \operatorname{Int}\left(P\left(\mathfrak{a}^{c /(q+1)}\right)\right)$. Again by Theorem 1.1] this implies that $\operatorname{lc}(R, \mathfrak{a}) \leq c /(q+1)$. Therefore, applying Theorem 1.1 from dFEM1 to $\mathfrak{a}$, we get (2.1).

Now we consider the general case. By (1.2) and (1.1), it is enough to prove that

$$
\operatorname{Vol}\left(\mathbb{R}_{+}^{n} \backslash P\left(\mathfrak{a}^{c}\right)\right) \geq \frac{(n+k)^{n}}{n !} .
$$

In order to obtain this bound on the volume, we replace $P\left(\mathfrak{a}^{c}\right)$ by a symmetric subset of $\mathbb{R}_{+}^{n}$ with a smaller complement. Let $S_{n}$, the symmetric group of $n$ letters, act in the obvious way on $\mathbb{R}_{+}^{n}$, and define

$$
Q:=\frac{1}{n !}\left(\underset{\sigma \in S_{n}}{\star} \sigma P\left(\mathfrak{a}^{c}\right)\right) .
$$

Clearly, $Q$ is an $S_{n}$-invariant subset of $\mathbb{R}_{+}^{n}$.

Lemma 3.2. $Q$ is convex, and $\operatorname{Vol}\left(\mathbb{R}_{+}^{n} \backslash Q\right) \leq \operatorname{Vol}\left(\mathbb{R}_{+}^{n} \backslash P\left(\mathfrak{a}^{c}\right)\right)$.

Proof. For short, let us denote $P\left(\mathfrak{a}^{c}\right)$ by $P$. By recursively applying Proposition 3.1 . we see that $\star_{\sigma \in S_{n}} \sigma P$ is convex; hence $Q$ is convex. To prove the second part of the lemma, we fix spherical coordinates $(\theta, \rho)$ on $\mathbb{R}_{+}^{n}$ with $\theta=\left(\theta_{1}, \ldots, \theta_{n-1}\right)$ and $0 \leq \theta_{j} \leq \pi / 2$. We denote by $\mathbf{u}(\theta, \rho)$ the vector with spherical coordinates $(\theta, \rho)$. Conversely, for a vector $\mathbf{u} \in \mathbb{R}_{+}^{n}$, we denote by $\theta(\mathbf{u})$ and $\rho(\mathbf{u})$ the multi-angle and length of $\mathbf{u}$. For any $\theta \in[0, \pi / 2]^{n-1}$ and $\sigma \in S_{n}$, we define $r_{\sigma}(\theta):=\inf \{\rho \mid \mathbf{u}(\theta, \rho) \in$ $\sigma P\}$ and $r(\theta):=\inf \{\rho \mid \mathbf{u}(\theta, \rho) \in Q\}$. By the definition of $Q$, we have

$$
r(\theta)=\frac{1}{n !} \sum_{\sigma \in S_{n}} r_{\sigma}(\theta)
$$

Let $V=\left\{\mathbf{u} \in \mathbb{R}_{+}^{n} \mid u_{1} \geq \cdots \geq u_{n} \geq 0\right\}$. This is a subcone of $\mathbb{R}_{+}^{n}$, and $\mathbb{R}_{+}^{n}$ is the union of the cones $\sigma V$ as $\sigma$ varies in $S_{n}$. Moreover, this union is almost-everywhere disjoint (the overlapping only occurs along the boundaries of the cones $\sigma V$ ), and we clearly have $\operatorname{Vol}(\sigma V \backslash Q)=\operatorname{Vol}(V \backslash Q)$ and $\operatorname{Vol}(\sigma V \backslash P)=\operatorname{Vol}\left(V \backslash \sigma^{-1} P\right)$ for 
every $\sigma \in S_{n}$. Then, denoting $\Theta=\left\{\theta \in[0, \pi / 2]^{n-1} \mid \mathbf{u}(\theta, 1) \in V\right\}$, we have

$$
\begin{aligned}
\operatorname{Vol}\left(\mathbb{R}_{+}^{n} \backslash Q\right) & =n ! \operatorname{Vol}(V \backslash Q)=n ! \int_{\Theta} \int_{0}^{r(\theta)} \rho^{n-1} \prod\left(\cos \theta_{i}\right)^{i-1} d \rho d \theta \\
& =\int_{\Theta}\left(n ! \frac{r(\theta)^{n}}{n}\right) \prod\left(\cos \theta_{i}\right)^{i-1} d \theta
\end{aligned}
$$

and

$$
\begin{aligned}
\operatorname{Vol}\left(\mathbb{R}_{+}^{n} \backslash P\right) & =\sum_{\sigma \in S_{n}} \operatorname{Vol}(V \backslash \sigma P)=\sum_{\sigma \in S_{n}} \int_{\Theta} \int_{0}^{r_{\sigma}(\theta)} \rho^{n-1} \prod\left(\cos \theta_{i}\right)^{i-1} d \rho d \theta \\
& =\int_{\Theta}\left(\sum_{\sigma \in S_{n}} \frac{r_{\sigma}(\theta)^{n}}{n}\right) \prod\left(\cos \theta_{i}\right)^{i-1} d \theta .
\end{aligned}
$$

Therefore, after we substitute the expression of $\rho(\theta)$ as given in (3.3) in the integral computing $\operatorname{Vol}\left(\mathbb{R}_{+}^{n} \backslash Q\right)$, the stated inequality between the two volumes is implied by the following numerical property: For any positive integer $n$ and positive real numbers $a_{1}, \ldots, a_{d}$, we have $d^{n-1} \sum_{j=1}^{d} a_{j}^{n} \geq\left(\sum_{j=1}^{d} a_{j}\right)^{n}$. A simple way to verify this property is to define $F(\mathbf{z})=d^{n-1} \sum_{j=1}^{d} z_{j}^{n}-\left(\sum_{j=1}^{d} z_{j}\right)^{n}$ and, denoting by $\mathbf{z}_{h}$ the vector with $j$-component equal to $a_{j}$ if $j<h$ and to $a_{h}$ if $j \geq h$, use induction on $h$ to show that $F\left(\mathbf{z}_{h}\right) \geq 0$ for every $h \in\{1, \ldots, d\}$.

Back to the proof of Theorem 2.1, let us write $k=n q+r$, with $0 \leq r \leq n-1$ (if $r=0$, then we are in the situation previously considered, so one may assume $r>0)$. Let $J$ vary among all subsets of $\{1, \ldots, n\}$ of cardinality $r$. For any such $J$, let $\mathbf{u}_{J}=\left(u_{J, i}\right)$ be the vector whose component $u_{J, i}$ equals $q+2$ if $i \in J$, and $q+1$ if $i \notin J$. Note that $\mathbf{u}_{J}-\mathbf{e}$ is in $\mathbb{N}^{n}$ and has one-norm $\left|\mathbf{u}_{J}-\mathbf{e}\right|=n q+r<k+1$, hence, in view of (3.1),

$$
\mathbf{x}^{\mathbf{u}_{J}-\mathbf{e}} \notin \mathcal{I}\left(\mathfrak{a}^{c}\right) .
$$

Therefore, by Theorem 1.1, $\mathbf{u}_{J} \notin \operatorname{Int}\left(P\left(\mathfrak{a}^{c}\right)\right)$ for every $J$. Then, since the set $\left\{\mathbf{u}_{J}|J \subset\{1, \ldots, n\}| J \mid,=r\right\}$ is $S_{n}$-invariant, we have $\mathbf{u}_{J} \notin \operatorname{Int}\left(\sigma P\left(\mathfrak{a}^{c}\right)\right)$ for every $J$ and every $\sigma \in S_{n}$, hence

$$
n ! \mathbf{u}_{J} \notin \underset{\sigma \in S_{n}}{\star} \operatorname{Int}\left(\sigma P\left(\mathfrak{a}^{c}\right)\right) \quad \text { for every } J .
$$

In conclusion, $\mathbf{u}_{J} \notin \operatorname{Int}(Q)$ for every $J$.

From now on, we fix $J=\{1, \ldots, r\}$. By the convexity of $Q$, we can find a hyperplane $H$, not containing the origin of $\mathbb{R}_{+}^{n}$, such that

$$
\mathbf{u}_{J} \in H \quad \text { and } \quad Q \subseteq H^{+} .
$$

We can furthermore assume that $H$ is invariant under the action of the stabilizer of $J$. Then $H$ has equation of the form

$$
\sum_{i=1}^{r} \frac{u_{i}}{a}+\sum_{i=r+1}^{n} \frac{u_{i}}{b}=1
$$

Note that $a$ and $b$ are positive numbers, since $\mathbb{R}_{+}^{n} \backslash Q$ is bounded, and we actually have

$$
b=\frac{a(n-r)(q+1)}{a-r(q+2)}
$$

by the first condition in (3.4). 
Suppose for the moment that $a \leq n+k$. It is easy to see that, if $a=n+k$, then $H$ intersects the ray spanned by $\mathbf{e}$ at the point $((n+k) / n) \mathbf{e}$. On the other hand, the value of the left-hand side of (3.5) at a point $\delta \mathbf{e}$ is equal to $\delta(1-r / a) /(q+1)$, and this is an increasing function of $a$. Then, using the inequality between the arithmetic mean and the geometric mean of the coefficients appearing in the left-hand side of (3.5), we easily obtain

$$
\operatorname{Vol}\left(\mathbb{R}^{n} \cap H^{-}\right)=\frac{a^{r} b^{n-r}}{n !} \geq \frac{(n+k)^{n}}{n !} .
$$

Since $Q \subseteq H^{+}$, this implies (3.2) when $a \leq n+k$. Thus, we can henceforth assume that $a \geq n+k$.

Let $V \subset \mathbb{R}_{+}^{n}$ be defined as in the proof of Lemma 3.2. We remark that

$$
\operatorname{Vol}\left(\mathbb{R}_{+}^{n} \backslash Q\right)=n ! \operatorname{Vol}(V \backslash Q) \text { and } \operatorname{Vol}(V \backslash Q) \geq \operatorname{Vol}\left(V \cap H^{-}\right)
$$

Then, in view of Lemma 3.2, in order to prove (3.2) it suffices to show that

$$
n ! \operatorname{Vol}\left(V \cap H^{-}\right) \geq \frac{(n+k)^{n}}{n !} .
$$

The left-hand side depends on $H$, that is, on $a$ and $b$, hence it becomes a function $f(a)$ of $a$ after we substitute the expression given in (3.6) for $b$. This function can be computed as follows. With the help of the linear transformation $T: \mathbb{R}^{n} \rightarrow \mathbb{R}^{n}$ given by $u_{i}=\frac{w_{i}}{i}+\cdots+\frac{w_{n}}{n}$ for $i=1, \ldots, n$, we compute

$$
n ! \operatorname{Vol}\left(V \cap H^{-}\right)=\frac{1}{n !} a^{r} \prod_{i=r+1}^{n}\left(\frac{r}{a i}+\frac{i-r}{b i}\right)^{-1} .
$$

Substituting (3.6) for $b$, we have

$$
\left(\frac{r}{a i}+\frac{i-r}{b i}\right)^{-1}=\frac{i(n-r)(q+1)}{i-r} \cdot \frac{a}{a+K_{i}},
$$

where

$$
K_{i}=\frac{r(n-r)(q+1)}{i-r}-r(q+2)
$$

Therefore we obtain

$$
f(a)=C a^{r} \prod_{i=r+1}^{n} \frac{a}{a+K_{i}},
$$

where $C$ is a positive constant depending on $n$ and $k$. Deriving, we get

$$
f^{\prime}(a)=\left(r+\sum_{i=r+1}^{n} \frac{K_{i}}{a+K_{i}}\right) C a^{r-1} \prod_{j=r+1}^{n} \frac{a}{a+K_{j}} .
$$

It is enough to focus on the first factor in the expression of $f^{\prime}(a)$. We observe that $K_{i}$ get smaller as $i$ increases, and that $K_{n}=-r$; hence $K_{i} \geq-r$ for $r+1 \leq i \leq n$. Moreover, the expression $K_{i} /\left(a+K_{i}\right)$ is an increasing function of $K_{i}$ for $K_{i}>-a$ (note that this inequality is always satisfied). Then it is easy to see that $f^{\prime}(a)>0$ for $a>n+k$. Since $f(n+k)=(n+k)^{n} / n$ !, we conclude that $f(a)>(n+k)^{n} / n$ ! for any such $a$. This completes the proof of the theorem. 
Remark 3.3. As suggested to the author by Howard Thompson, one can also seek a proof of Theorem 2.1 by using a Veronese embedding of $R$, so to force the divisibility of $k$. This approach requires the extension of the main result of dFEM1 to certain singular rings, which is possible by the generalization of Howald's theorem to singular Gorenstein toric rings (for such generalization, see [Bli] or [HY]). We prefer to work with regular rings, as this facilitates the extension of the proof of Theorem 2.1 to the analogous result in positive characteristic (see Section 5 below).

Proof of Theorem 2.3. As the proof is very similar to the one of dFEM1, Theorem 1.4, we will just give a brief outline, explaining the necessary modifications. One direction is clear, so we can focus on the proof of the "only if" part.

We can reduce to the case in which $R$ is the localization at the origin of a polynomial ring. Assuming that $e(\mathfrak{a})=(n+k)^{n} / c^{n}$, we easily observe that $(n+k) / c \in \mathbb{N}$. Then the theorem will follow if we show that $\mathfrak{a} \subseteq \mathfrak{m}^{(n+k) / c}$.

We deform all the powers $\mathfrak{a}^{r}$ to monomial ideals in $\left(\mathfrak{a}^{r}\right)$, as follows. We first deform $\mathfrak{a}^{r}$ to the tangent cone, and then take the initial ideal of the resulting ideal with respect to a fixed monomial order. In particular, doing it this way, we have $\mathfrak{a} \subseteq \mathfrak{m}^{(n+k) / c}$ if and only if $\operatorname{in}(\mathfrak{a}) \subseteq \mathfrak{m}^{(n+k) / c}$. Since we are assuming that $e(\mathfrak{a})=(n+k)^{n} / c^{n}$, we have

$$
\lim _{m \rightarrow \infty} \frac{n ! \ell\left(R / \operatorname{in}\left(\mathfrak{a}^{m}\right)\right)}{m^{n}}=\lim _{m \rightarrow \infty} \frac{n ! \ell\left(R / \mathfrak{a}^{m}\right)}{m^{n}}=e(\mathfrak{a})=\frac{(n+k)^{n}}{c^{n}} .
$$

Set $P_{m}=\frac{1}{m} P\left(\operatorname{in}\left(\mathfrak{a}^{m}\right)\right)$ and $P_{\infty}=\bigcup_{s \in \mathbb{N}} P_{2^{s}}$. We see from (1.3) that the volume of $\mathbb{R}_{+}^{n} \backslash P_{m}$ is asymptotic to $\ell\left(R / \operatorname{in}\left(\mathfrak{a}^{m}\right)\right) / m^{n}$ for $m \rightarrow \infty$. Observing that $P_{2^{s}} \subseteq P_{2^{t}}$ if $s<t$, we conclude that $P_{\infty}$ is convex and

$$
n ! \operatorname{Vol}\left(\mathbb{R}_{+}^{n} \backslash P_{\infty}\right)=\frac{(n+k)^{n}}{c^{n}} .
$$

By Proposition 1.2, we have $\mathcal{J}\left(R, \operatorname{in}(\mathfrak{a})^{c}\right) \subseteq \mathfrak{m}^{k+1}$. Then, using the inclusion $\operatorname{in}(\mathfrak{a})^{m} \subseteq \operatorname{in}\left(\mathfrak{a}^{m}\right)$, we get $\mathcal{J}\left(R, \operatorname{in}\left(\mathfrak{a}^{m}\right)^{c / m}\right) \subseteq \mathfrak{m}^{k+1}$. Therefore, arguing as in the proof of Theorem 2.1 and using the notation introduced there, we see that $\mathbf{u}_{J} \notin$ Int $P\left(\operatorname{in}\left(\mathfrak{a}^{m}\right)^{c / m}\right)=\operatorname{Int}\left(c P_{m}\right)$ for every $J$. Passing to the limit for $m=2^{s} \rightarrow \infty$, we conclude that $\mathbf{u}_{J} \notin \operatorname{Int}\left(c P_{\infty}\right)$. Then, by the same arguments used in the proof of Theorem 2.1, applied this time to $c P_{\infty}$ in place of $P\left(\mathfrak{a}^{c}\right)$, one can check that (3.7) can only be satisfied if

$$
\frac{n+k}{n} \cdot \mathbf{e} \notin \operatorname{Int}\left(c P_{\infty}\right)
$$

After this observation, we can conclude as in the proof of [dFEM1, Theorem 1.4.

\section{A general formula ON LENGTHS IN SMALL DimENSiON}

We expect that the inequalities stated in Theorems 2.1 and 2.2 are particular cases of more general formulae, at least when $c=1$. We show that this is the case for the second theorem if the dimension of $R$ is at most 3 .

Theorem 4.1. Let $\mathfrak{a}$ be an $\mathfrak{m}$-primary ideal of a regular local ring $(R, \mathfrak{m})$ of dimension $n \leq 3$, essentially of finite type over a field of characteristic zero. Assume that $\mathcal{I}(\mathfrak{a})$ is not trivial. Then

$$
\ell(R / \mathfrak{a}) \geq \ell\left(R / \mathcal{I}(\mathfrak{a}) \mathfrak{m}^{n-1}\right) .
$$


Remark 4.2. Since $\mathcal{I}(\overline{\mathfrak{a}})=\mathcal{I}(\mathfrak{a})$, the statement above can be strengthened by replacing $\ell(R / \mathfrak{a})$ by $\ell(R / \overline{\mathfrak{a}})$ in the left-hand side of (4.1).

Corollary 4.3. Keeping the notation as in Theorem 4.1, suppose that $\mathcal{I}(\mathfrak{a}) \subseteq \mathfrak{m}^{k+1}$ for some $k \in \mathbb{N}$. Then

$$
\ell(R / \mathfrak{a}) \geq \ell\left(R / \mathfrak{m}^{n+k}\right)=\left(\begin{array}{c}
2 n+k-1 \\
n
\end{array}\right) .
$$

Remark 4.4. Since the binomial on the right-hand side of (4.2) is strictly larger than $(n+k)^{n} / n$ ! if $n \geq 2$, Corollary 4.3 gives a more precise bound on the length than Theorem 2.1 when $n \in\{2,3\}$ and $c=1$. Of course this improvement is lost in the limit computing the Samuel multiplicity of $\mathfrak{a}$ (in fact we know that the statement of Theorem 2.2 is sharp).

Before passing to the proof of Theorem 4.1 we give a few comments on inequality (4.1). Such an inequality looks very similar to another inequality, namely

$$
\ell(R / \mathfrak{a}) \geq \ell\left(R / \mathcal{I}\left(\mathfrak{a m}^{n-1}\right)\right) .
$$

It should be clear that (4.3) is actually a weaker inequality, merely following an inclusion of ideals. To see this, let $X=\operatorname{Spec} R$, let $f: X^{\prime} \rightarrow X$ be a log resolution of $(R, \mathfrak{a})$, and write $f^{-1} \mathfrak{m} \cdot \mathcal{O}_{X^{\prime}}=\mathcal{O}_{X^{\prime}}(-F)$ and $f^{-1} \mathfrak{a} \cdot \mathcal{O}_{X^{\prime}}=\mathcal{O}_{X^{\prime}}(-E)$. Since $\left(R, \mathfrak{m}^{n-1}\right)$ is a canonical pair, the divisor $K_{f}-(n-1) F$ is effective. Thus, we have

$$
\mathfrak{a} \subseteq \overline{\mathfrak{a}}=f_{*} O_{X^{\prime}}(-E) \subseteq f_{*} O_{X^{\prime}}\left(K_{f}-E-(n-1) F\right)=\mathcal{I}\left(\mathfrak{a m}^{n-1}\right) .
$$

This gives (4.3). On the contrary, the example below shows that (4.1) does not follow, in general, by an inclusion of ideals.

Example 4.5. Let $\mathfrak{a}$ be the ideal $\left(x^{5}, y^{4}, z^{2}\right)$ of $R=k[x, y, z]$ (or its integral closure). Then $\mathcal{I}(\mathfrak{a})$ is non-trivial, and $\mathfrak{a} \nsubseteq \mathcal{I}(\mathfrak{a}) \mathfrak{m}^{2}$, since $z^{2} \notin \mathcal{I}(\mathfrak{a}) \mathfrak{m}^{2}$. Nevertheless, it is easy to verify that $\ell(R / \mathfrak{a}) \geq \ell\left(R / \mathcal{I}(\mathfrak{a}) \mathfrak{m}^{2}\right)$, as we know it must be by Theorem 4.1 .

The next lemma will be used to reduce the proof of Theorem 4.1 to the case of monomial ideals.

Lemma 4.6. Let $R=k\left[x_{1}, \ldots, x_{n}\right]_{\mathfrak{m}}$, with $\mathfrak{m}=\left(x_{1}, \ldots, x_{n}\right)$. Then, to prove a statement of the form

$$
\ell\left(R / \mathfrak{b}_{1}\right) \geq \ell\left(R / \mathcal{I}\left(\mathfrak{b}_{2}\right) \mathfrak{b}_{3}\right)
$$

where $\mathfrak{b}_{i}$ are $\mathfrak{m}$-primary ideals of $R$, it is enough to show that, after computing initial ideals with respect to some monomial order on $R$,

$$
\ell\left(R / \operatorname{in}\left(\mathfrak{b}_{1}\right)\right) \geq \ell\left(R / \mathcal{I}\left(\operatorname{in}\left(\mathfrak{b}_{2}\right)\right) \operatorname{in}\left(\mathfrak{b}_{3}\right)\right) .
$$

Proof. We have

$$
\mathcal{I}\left(\operatorname{in}\left(\mathfrak{b}_{2}\right)\right) \operatorname{in}\left(\mathfrak{b}_{3}\right) \subseteq \operatorname{in}\left(\mathcal{I}\left(\mathfrak{b}_{2}\right)\right) \operatorname{in}\left(\mathfrak{b}_{3}\right) \subseteq \operatorname{in}\left(\mathcal{I}\left(\mathfrak{b}_{2}\right) \mathfrak{b}_{3}\right),
$$

where the first inclusion follows from Proposition 1.2 , and the second one is obvious. We get $\ell\left(R / \mathcal{I}\left(\operatorname{in}\left(\mathfrak{b}_{2}\right)\right)\right.$ in $\left.\left(\mathfrak{b}_{3}\right)\right) \geq \ell\left(R / \operatorname{in}\left(\mathcal{I}\left(\mathfrak{b}_{2}\right) \mathfrak{b}_{3}\right)\right)$. On the other hand, we have $\ell\left(R / \mathfrak{b}_{1}\right)=\ell\left(R / \operatorname{in}\left(\mathfrak{b}_{1}\right)\right)$ and $\ell\left(R / \mathcal{I}\left(\mathfrak{b}_{2}\right) \mathfrak{b}_{3}\right)=\ell\left(R / \operatorname{in}\left(\mathcal{I}\left(\mathfrak{b}_{2}\right) \mathfrak{b}_{3}\right)\right)$ by flatness.

Proof of Theorem 4.1. We reduce to the case $R=k\left[x_{1}, \ldots, x_{n}\right]_{\mathfrak{m}}$, with $\mathfrak{m}=\left(x_{1}, \ldots\right.$, $x_{n}$ ), as in the proof of Theorem 2.1, and fix a monomial order inducing a flat degeneration of the ideals $\mathfrak{a}, \mathcal{I}(\mathfrak{a})$ and $\mathcal{I}(\mathfrak{a}) \mathfrak{m}^{n-1}$ to their initial ideals $\operatorname{in}(\mathfrak{a}), \operatorname{in}(\mathcal{I}(\mathfrak{a}))$ and $\operatorname{in}\left(\mathcal{I}(\mathfrak{a}) \mathfrak{m}^{n-1}\right)$. By the semi-continuity of the multiplier ideal, $\mathcal{I}(\operatorname{in}(\mathfrak{a})) \neq R$ if $\mathcal{I}(\mathfrak{a}) \neq R$. Therefore in $(\mathfrak{a})$ satisfies the assumption of the theorem. Then, by 
Lemma 4.6, it is enough to show that (4.1) holds for in $(\mathfrak{a})$ in place of $\mathfrak{a}$. So, we can assume that $\mathfrak{a}$ is a monomial ideal.

The theorem is trivial if $n=1$; we will independently discuss the two cases when $n$ is 2 or 3 . Suppose that $n=2$. Fix $\mathbf{u} \in \mathbb{N}^{2}$ such that $\mathbf{x}^{\mathbf{u}} \in \mathfrak{a}$. Note that $\mathbf{u} \neq(0,0)$, since $\mathfrak{a} \neq R$. Thus there is a choice of $i \in\{1,2\}$ for which $\mathbf{u}+\mathbf{e}_{i} \in \operatorname{Int}(P(\mathfrak{a}))$. This gives

$$
\mathbf{x}^{\mathbf{u}+\mathbf{e}_{i}-\mathbf{e}} \in \mathcal{I}(\mathfrak{a})
$$

by Theorem 1.1. Then, taking $\{i, j\}=\{1,2\}$, we get

$$
\mathbf{x}^{\mathbf{u}}=\mathbf{x}^{\mathbf{u}+\mathbf{e}_{i}-\mathbf{e}} \cdot \mathbf{x}^{\mathbf{e}_{j}} \in \mathcal{I}(\mathfrak{a}) \mathfrak{m} .
$$

We conclude that $\mathfrak{a} \subseteq \mathcal{I}(\mathfrak{a}) \mathfrak{m}$, and this obviously implies the inequality between the lengths.

Assume now that $n=3$, and fix $\mathbf{u}=\left(u_{1}, u_{2}, u_{3}\right) \in \mathbb{N}^{3}$ such that $\mathbf{x}^{\mathbf{u}} \in \mathfrak{a}$. Note that $\mathbf{u} \neq(0,0,0)$. If $\#\left\{i \mid u_{i}=0\right\} \leq 1$, then essentially the same arguments used for the two-dimensional case give $\mathbf{x}^{\mathbf{u}} \in \mathcal{I}(\mathfrak{a}) \mathfrak{m}^{2}$. It remains to analyze the case when $\#\left\{i \mid u_{i}=0\right\}=2$. For each $i \in\{1,2,3\}$, let

$$
a_{i}=\min \left\{a \in \mathbb{N} \mid x_{i}^{a} \in \mathfrak{a}\right\} .
$$

Since $\mathcal{I}(\mathfrak{a}) \subseteq \mathfrak{m}$, we have $a_{i} \geq 2$ for $i=1,2,3$. We can assume that $a_{1} \geq a_{2} \geq a_{3}$. Let $H \subset \mathbb{R}_{+}^{3}$ be the plane defined by $\sum u_{i} / a_{i}=1$. Note that $\operatorname{Int}\left(H^{+}\right) \subseteq \operatorname{Int}(P(\mathfrak{a}))$. We consider the vectors

$$
\mathbf{v}_{i}:=\left(a_{i}-1\right) \mathbf{e}_{i}+\mathbf{e}_{j}+\mathbf{e}_{k}, \quad\{i, j, k\}=\{1,2,3\} .
$$

Since

$$
\frac{a_{1}-1}{a_{1}}+\frac{1}{a_{2}}+\frac{1}{a_{3}}=1-\frac{1}{a_{1}}+\frac{1}{a_{2}}+\frac{1}{a_{3}}>1,
$$

$\mathbf{v}_{1}$ is in $\operatorname{Int}\left(H^{+}\right)$, and so in $\operatorname{Int}(P(\mathfrak{a}))$. Then

$$
x_{1}^{a_{1}-2}=\mathbf{x}^{\mathbf{v}_{1}-\mathbf{e}} \in \mathcal{I}(\mathfrak{a}),
$$

hence $x_{1}^{a_{1}} \in \mathcal{I}(\mathfrak{a}) \mathfrak{m}^{2}$. In particular, we have that $x_{1}^{a} \in \mathcal{I}(\mathfrak{a}) \mathfrak{m}^{2}$ for every $a \geq a_{1}$. Similarly, we see that $x_{2}^{a} \in \mathcal{I}(\mathfrak{a}) \mathfrak{m}^{2}$ for every $a \geq a_{2}$. For $i=3$, there are two possibilities. If $1 / a_{3}<1 / a_{1}+1 / a_{2}$, then the previous arguments apply to this case too, giving $x_{3}^{a} \in \mathcal{I}(\mathfrak{a}) \mathfrak{m}^{2}$ for every $a \geq a_{3}$, hence $\mathfrak{a} \subseteq \mathcal{I}(\mathfrak{a}) \mathfrak{m}^{2}$. However, if

$$
\frac{1}{a_{3}} \geq \frac{1}{a_{1}}+\frac{1}{a_{2}}
$$

then we can only conclude that $x_{3}^{a} \in \mathcal{I}(\mathfrak{a}) \mathfrak{m}^{2}$ for $a \geq a_{3}+1$. In other words the monomial $x_{3}^{a_{3}}$, that we know belongs to $\mathfrak{a}$, may be not contained in $\mathcal{I}(\mathfrak{a}) \mathfrak{m}^{2}$. We remark that this is the only monomial in $\mathfrak{a}$ that does not necessarily belong to $\mathcal{I}(\mathfrak{a}) \mathfrak{m}^{2}$. On the other hand, (4.4) implies

$$
\frac{a_{1}-2}{a_{1}}+\frac{1}{a_{2}}+\frac{1}{a_{3}} \geq 1-\frac{2}{a_{1}}+\frac{1}{a_{2}}+\frac{1}{a_{1}}+\frac{1}{a_{2}}>1 .
$$

Note also that $a_{1} \geq 2 a_{3} \geq 4$ by (4.4). Then $\left(a_{1}-2\right) \mathbf{e}_{1}+\mathbf{e}_{2}+\mathbf{e}_{3} \in \operatorname{Int}(P(\mathfrak{a}))$, hence

$$
x_{1}^{a_{1}-3} \in \mathcal{I}(\mathfrak{a}) .
$$

Therefore $x_{1}^{a_{1}-1}$, which is not an element of $\mathfrak{a}$, is contained in $\mathcal{I}(\mathfrak{a}) \mathfrak{m}^{2}$. In conclusion, the number of monomials that are not contained in $\mathfrak{a}$ is not smaller than the number of those that are not in $\mathcal{I}(\mathfrak{a}) \mathfrak{m}^{2}$. This precisely means that $\ell(R / \mathfrak{a}) \geq \ell\left(R / \mathcal{I}(\mathfrak{a}) \mathfrak{m}^{2}\right)$. 
Remark 4.7. We suspect it might be possible to extend Theorem 4.1 to all dimensions by a suitable count of "gains and losses" similarly to the way done in the proof for the case $n=3$.

\section{Analogous Results in positive characteristic}

Let $R$ be a commutative Noetherian ring, with identity, of characteristic $p>0$. We will use the letter $q$ to denote a power $p^{e}$ of $p$. For any $e \in \mathbb{N}$, we denote by ${ }^{e} R$ the ring $R$ regarded as an $R$-module via the $e$-times iterated Frobenius map $F^{e}: R \rightarrow R$. For an $R$-module $M$, we define $\mathbb{F}_{R}^{e}(M):={ }^{e} R \otimes_{R} M$, and regard it as an $R$-module by the action of $R={ }^{e} R$ from the left. There is an induced $e$-times iterated Frobenius map $F^{e}: M \rightarrow \mathbb{F}_{R}^{e}(M)$. We denote by $z^{q} \in \mathbb{F}_{R}^{e}(M)$ the image by this map of an element $z \in M$. If $N$ is an $R$-submodule of $M$, then $N_{M}^{[q]}$ will denote the image of the induced map $\mathbb{F}^{e}(N) \rightarrow \mathbb{F}^{e}(M)$. For instance, if $I$ is an ideal of $R$, then $I_{R}^{[q]}$ is the ideal of $R$ generated by the $q$-powers of the elements of $I$.

Consider an ideal $\mathfrak{a}$ of $R$ (we will always assume that $\mathfrak{a}$ contains a non-zero divisor of $R$ ), and fix a positive rational number $c$. Associated to $\mathfrak{a}$ and $c$, Hara and Yoshida have introduced in $[\mathrm{HY}]$ a new invariant of tight closure, the definition of which we now recall.

Definition 5.1 (Hara-Yoshida). Let $N \subseteq M$ be $R$-modules. Given a rational number $c>0$, the $\mathfrak{a}^{c}$-tight closure $N_{M}^{* \mathfrak{a}^{c}}$ of $N$ in $M$ is defined to be the submodule of $M$ consisting of all elements $z$ for which there exists an element $b \in R$, not contained in any minimal prime ideal, such that $b z^{q} \mathfrak{a}^{[c q]} \subseteq N_{M}^{[q]}$ for all $q=p^{e} \gg 0$.

For the main properties of this operation, we refer to $[\mathrm{HY}$. We limit ourselves to recall that, if $\mathfrak{a}=R$, then the $R^{c}$-tight closure $N_{M}^{* R^{c}}$ is nothing but the usual tight closure $N_{M}^{*}$ introduced by Hochster and Huneke in $[\mathrm{HH}]$.

In $[\mathrm{HY}$, the above more general notion of tight closure is used to define a new ideal associated to $\mathfrak{a}$ and $c$. This is denoted by $\tau\left(\mathfrak{a}^{c}\right)$, and is a generalization of the test ideal introduced in $[\mathrm{HH}]$.

Definition 5.2 (Hara-Yoshida). Let $E=\bigoplus_{\mathfrak{m}} E_{R}(R / \mathfrak{m})$ be the direct sum, taken over all maximal ideals $\mathfrak{m}$ of $R$, of the injective envelopes of the residue fields $R / \mathfrak{m}$. Then we define

$$
\tau\left(\mathfrak{a}^{c}\right):=\bigcap_{M \subseteq E} \operatorname{Ann}_{R}\left(0_{M}^{* \mathfrak{a}^{c}}\right),
$$

where $M$ runs through all finitely generated $R$-submodules of $E$.

We now collect a few properties of these ideals. For simplicity, we henceforth assume that $R$ is a finite-dimensional regular ring essentially of finite type over a perfect field $k$ of characteristic $p>0$. We refer the reader to [HY] and [HT] for the precise statements in full generality, and for other properties as well.

By assuming that $R$ is regular, already the definition of the ideal $\tau\left(\mathfrak{a}^{c}\right)$ becomes more manageable, since in this case Theorem 1.13 in $[\mathrm{HY}]$ gives

$$
\tau\left(\mathfrak{a}^{c}\right)=\operatorname{Ann}_{R}\left(0_{E}^{* \mathfrak{a}^{c}}\right) .
$$

Hara and Yoshida also prove that, if $\mathfrak{a}$ is reduced from characteristic zero to characteristic $p \gg 0$ together with a $\log$ resolution, then $\tau\left(\mathfrak{a}^{c}\right)=\mathcal{I}\left(\mathfrak{a}^{c}\right)$ (see [HY], Theorem $6.8)$. More generally, the analogy between the two ideals is expressed by several similar properties they satisfy. 
It follows from the definition and the fact that $E_{R}(R / \mathfrak{m})=E_{R_{\mathfrak{m}}}\left(R_{\mathfrak{m}} / \mathfrak{m} R_{\mathfrak{m}}\right)$ that $\tau\left(\left(\mathfrak{a} R_{\mathfrak{m}}\right)^{c}\right)=\tau\left(\mathfrak{a}^{c}\right) R_{\mathfrak{m}}$ for any maximal ideal $\mathfrak{m}$ of $R$. Furthermore, it is shown in [HT], Proposition 3.2, that, if $(R, \mathfrak{m})$ is a local ring and $\widehat{R}$ is its $\mathfrak{m}$-adic completion, then $\tau\left((\mathfrak{a} \widehat{R})^{c}\right)=\tau\left(\mathfrak{a}^{c}\right) \widehat{R}$. Bearing these things in mind, we can state the next properties without assuming that $R$ is a (complete) local ring.

The ideal $\tau\left(\mathfrak{a}^{c}\right)$ behaves similarly to multiplier ideals with respect to restrictions: if $x$ is a non-zero element of $R$ and $S:=R / x R$ is normal, then $\tau\left((\mathfrak{a} S)^{c}\right) \subseteq \tau\left(\mathfrak{a}^{c}\right) S$. Moreover, if $R$ is a polynomial ring (or its localization at the origin) and $\mathfrak{a}$ is a monomial ideal, then the multiplier ideal of $\mathfrak{a}^{c}$ is defined, and $\tau\left(\mathfrak{a}^{c}\right)=\mathcal{I}\left(\mathfrak{a}^{c}\right)$. In particular, $\tau\left(\mathfrak{a}^{c}\right)$ is a monomial ideal and, using notation as introduced in Section 1 , $\mathbf{x}^{\mathbf{u}} \in \tau\left(\mathfrak{a}^{c}\right)$ if and only if $\mathbf{u}+\mathbf{e} \in \operatorname{Int}\left(P\left(\mathfrak{a}^{c}\right)\right) \cap \mathbb{N}^{n}$. For these two properties, see [HY], Theorem 6.10. We also recall that, if $\overline{\mathfrak{a}}$ is the integral closure of $\mathfrak{a}$, then $\tau\left(\mathfrak{a}^{c}\right)=\tau\left(\overline{\mathfrak{a}}^{c}\right)$; this follows from $[\mathrm{HY}$, Proposition 1.3.

In order to establish positive characteristic analogues of the results proven in the previous sections of this paper, it is necessary to control ideals of the form $\tau\left(\mathfrak{a}^{c}\right)$ under degeneration to monomial ideals.

Proposition 5.3. Fix a perfect field $k$ of characteristic $p>0$, let $R=k\left[x_{1}, \ldots, x_{n}\right]$, and consider an ideal $\mathfrak{a}$ of $R$ together with a positive rational number $c$. Then, for any monomial order giving a flat degeneration of $\mathfrak{a}$ and $\tau\left(\mathfrak{a}^{c}\right)$ to their initial ideals $\operatorname{in}(\mathfrak{a})$ and $\operatorname{in}\left(\tau\left(\mathfrak{a}^{c}\right)\right)$, we have

$$
\tau\left(\operatorname{in}(\mathfrak{a})^{c}\right) \subseteq \operatorname{in}\left(\tau\left(\mathfrak{a}^{c}\right)\right) .
$$

The proof of Proposition 5.3 is very similar to that of Proposition 1.2 , and we will sketch it below. Before doing so, we only need to fix the following property.

Lemma 5.4. Let $R$ and $S$ be two finite-dimensional polynomial rings over a perfect field $k$ of characteristic $p>0$. Let $T=R \otimes S$. Then, for any ideal $\mathfrak{a}$ of $R$,

$$
\tau\left((\mathfrak{a} \otimes S)^{c}\right)=\tau\left(\mathfrak{a}^{c}\right) \otimes S
$$

Proof. We can check the equality locally, even passing to completion. Thus we may reduce to the case when $R$ and $S$ are formal power series rings and $T=R \widehat{\otimes} S$. In fact, it is enough to prove the proposition when $S=k[[t]]$, as we can then recursively apply the result to get the statement for any dimension of $S$.

The injective envelope of the residual fields of the rings $R, S$ and $T$ are respectively $E_{R}=k\left(x_{1}, \ldots, x_{n}\right) / k\left[x_{1}, \ldots, x_{n}\right], E_{S}=k(t) / k[t]$, and

$$
E_{T}=k\left(x_{1}, \ldots, x_{n}, t\right) / k\left[x_{1}, \ldots, x_{n}, t\right]=E_{R} \otimes E_{S} .
$$

Moreover, we have $\mathbb{F}^{e}\left(E_{T}\right)=\mathbb{F}^{e}\left(E_{R}\right) \otimes \mathbb{F}^{e}\left(E_{S}\right)$.

We have $\tau\left(\left((\mathfrak{a} \otimes S)^{c}\right) T\right)=\operatorname{Ann}_{T}\left(0_{E_{T}}^{*(\mathfrak{a} \otimes S)^{c}}\right)$ and

$$
\left(\tau\left(\mathfrak{a}^{c}\right) \otimes S\right) T=\left(\operatorname{Ann}_{R}\left(0_{E_{R}}^{* \mathfrak{a}^{c}}\right) \otimes E_{S}\right) T=\operatorname{Ann}_{T}\left(0_{E_{R}}^{* \mathfrak{a}^{c}} \otimes E_{S}\right) .
$$

Thus, to prove the lemma, it is enough to show that

$$
0_{E_{T}}^{*(\mathfrak{a} \otimes S)^{c}}=0_{E_{R}}^{* \mathfrak{a}^{c}} \otimes E_{S} .
$$

One easily sees that the right-hand side is contained in the left-hand side. To check the other inclusion, let $z \in 0_{E_{T}}^{*(\mathfrak{a} \otimes S)^{c}}$. Since $E_{S}=k(t) / k[t]$, we can write

$$
z=\sum_{i=1}^{m} w_{i} \otimes t^{-a_{i}}
$$


with $w_{i} \in E_{R}$ and $0<a_{1}<\cdots<a_{m}$. By (5.1), we have

$$
w_{i} \otimes t^{-a_{i}} \in 0_{E_{T}}^{*(\mathfrak{a} \otimes S)^{c}} \quad \text { for every } i .
$$

Observing that $(\mathfrak{a} \otimes S)^{\lceil c q\rceil}=\mathfrak{a}^{\lceil c q\rceil} \otimes S$ gives

$$
0=\left(w_{i}^{q} \otimes t^{-a_{i} q}\right)\left(\mathfrak{a}^{\lceil c q\rceil} \otimes S\right)=\left(w_{i}^{q} \mathfrak{a}^{\lceil c q\rceil}\right) \otimes\left(t^{-a_{i} q} S\right) \quad \text { in } \mathbb{F}^{e}\left(E_{T}\right)
$$

for every $i$ and all $q=p^{e} \gg 0$. Since $t^{-a_{i} q} S$ is never zero in $\mathbb{F}^{e}\left(E_{S}\right)$, we conclude that $w_{i}^{q} \mathfrak{a}^{\lceil c q\rceil}=0$ in $\mathbb{F}^{e}\left(E_{R}\right)$ for sufficiently large $q$. Therefore $w_{i} \in 0_{E_{R}}^{* \mathfrak{a}^{c}}$. This in turns yields $z \in 0_{E_{R}}^{* \mathfrak{a}^{c}} \otimes E_{S}$.

Proof of Proposition 5.3. As in the proof of Proposition 1.2, let

$$
\mathfrak{b} \subset T:=k\left[x_{1}, \ldots, x_{n}, t\right]
$$

be the ideal corresponding to the deformation of $\mathfrak{a}$, and consider the isomorphism from $T_{t}:=k\left[x_{1}, \ldots, x_{n}, t, t^{-1}\right]$ to $R \otimes k\left[t, t^{-1}\right]$ sending $\mathfrak{b} T_{t}$ to $\mathfrak{a} \otimes k\left[t, t^{-1}\right]$. Via this isomorphism, and by Lemma 5.4 , we have

$$
\tau\left(\left(\mathfrak{b} T_{t}\right)^{c}\right) \cong \tau\left(\mathfrak{a}^{c}\right) \otimes k\left[t, t^{-1}\right] .
$$

From here the proof proceeds as in the proof of Proposition 1.2

Finally, we can state analogous results as those proven in the previous sections for multiplier ideals. So, let $(R, \mathfrak{m})$ and be an $n$-dimensional regular local ring, essentially of finite type over a perfect field $k$ of positive characteristic, and consider an $\mathfrak{m}$-primary ideal $\mathfrak{a}$ of $R$.

Theorem 5.5. With the above notation, assume that $\tau\left(\mathfrak{a}^{c}\right) \subseteq \mathfrak{m}^{k+1}$ for some $c \in \mathbb{Q}_{+}$and $k \in \mathbb{N}$. Then

$$
\ell(R / \mathfrak{a}) \geq \frac{(n+k)^{n}}{n ! c^{n}},
$$

with strict inequality if $n \geq 2$, and

$$
e(\mathfrak{a}) \geq \frac{(n+k)^{n}}{c^{n}} .
$$

Moreover, equality holds in the last formula if and only if $(n+k) / c \in \mathbb{N}$ and the integral closure of $\mathfrak{a}$ is equal to $\mathfrak{m}^{(n+k) / c}$.

Theorem 5.6. With the above notation, assume that $R$ has dimension $n \leq 3$ and that $\tau(\mathfrak{a})$ is not trivial. Then

$$
\ell(R / \mathfrak{a}) \geq \ell\left(R / \tau(\mathfrak{a}) \mathfrak{m}^{n-1}\right) .
$$

The proof of these theorems is the same as those of the corresponding statements for multiplier ideals in characteristic zero.

\section{ACKNOWLEDGEMENTS}

The last section of this paper was conceived during my visit to the University of Tokyo. I thank Toshiyuki Katsura for his invitation and support, and Shunsuke Takagi for several enlightening discussions and remarks. Furthermore, I thank Lawrence Ein, Mel Hochster, Rob Lazarsfeld, and Howard Thompson for many comments and conversations that have been useful in the writing of this paper. Finally, I thank the referee for his suggestions and corrections. 


\section{REFERENCES}

[Bli] M. Blickle, Multiplier ideal and module on a toric variety, Math. Z. 248 (2004), no. 1, 113-121. MR.2092724

[dFEM1] T. de Fernex, L. Ein and M. Mustaţă, Multiplicities and log canonical threshold, J. Algebraic Geom. 13 (2004), 603-615. MR2047683 (2005b:14008)

[dFEM2] T. de Fernex, L. Ein and M. Mustaţă, Bounds on log canonical thresholds with application to birational rigidity, Math. Res. Lett. 10 (2003), 219-236. MR.1981899 (2004e:14060)

[Eis] D. Eisenbud, Commutative Algebra with a View toward Algebraic Geometry, Grad. Texts in Math. 150, Springer, New York, 1995. MR.1322960 (97a:13001)

[HY] N. Hara and K. Yoshida, A generalization of tight closure and multiplier ideals, Trans. Amer. Math. Soc. 355 (2003), 3143-3174. MR1974679 (2004i:13003)

[HT] N. Hara and S. Takagi, On a generalization of test ideals, Nagoya Math. J. 175 (2004), 59-74. MR 2085311

[HH] M. Hochster and C. Huneke, Tight closure, invariant theory and the Briançon-Skoda theorem, J. Amer. Math. Soc. 3 (1990), 21-116. MR.1017784 (91g:13010)

[How] J. Howald, Multiplier ideals of monomial ideals, Trans. Amer. Math. Soc. 353 (2001), 2665-2671. MR1828466 (2002b:14061)

[Laz] R. Lazarsfeld, Positivity in Algebraic Geometry, book in print.

[TW] S. Takagi and K.-i. Watanabe, On F-pure thresholds, J. Algebra 282 (2004), no. 1, 278-297. MR2097584

[Tei] B. Teissier, Monomes, volumes et multiplicités, in Introduction à la Théorie des Singularités, II, pp. 127-141, Travaux en Cours, 37, Hermann, Paris, 1988. MR.1074593 (92e:14003)

Department of Mathematics, University of Michigan, East Hall, 525 East University Avenue, Ann Arbor, Michigan 48109-1109

E-mail address: defernex@umich.edu

Current address: School of Mathematics, Institute for Advanced Study, 1 Einstein Drive, Princeton, New Jersey 08540

E-mail address: defernex@math.ias.edu 